\title{
Higher Order Local Accuracy by Averaging in the Finite Element Method
}

\author{
By J. H. Bramble and A. H. Schatz*
}

\begin{abstract}
Let $u_{h}$ be a Ritz-Galerkin approximation, corresponding to the solution $u$ of an elliptic boundary value problem, which is based on a uniform subdivision in the interior of the domain. In this paper we show that by "averaging" the values of $u_{h}$ in the neighborhood of a point $x$ we may (for a wide class of problems) construct an approximation to $u(x)$ which is often a better approximation than $u_{h}(x)$ itself. The "averaging" operator does not depend on the specific elliptic operator involved and is easily constructed.
\end{abstract}

1. Introduction. In this paper we shall discuss some local "superconvergence" results for a large class of Ritz-Galerkin methods, which are used to approximate solutions of elliptic boundary value problems. Briefly, let $\Omega$ be a bounded domain in $\mathbf{R}^{N}$ with boundary $\partial \Omega$ and for simplicity consider the boundary value problem

$$
\begin{aligned}
& L u=-\sum_{i, j=1}^{N} \frac{\partial}{\partial x_{j}}\left(a_{i j}(x) \frac{\partial u}{\partial x_{i}}\right)+\sum_{i=1}^{N} b_{i}(x) \frac{\partial u}{\partial x_{i}}+c(x) u=f \quad \text { in } \Omega, \\
& b u=g \text { on } \partial \Omega
\end{aligned}
$$

where $L$ is uniformly elliptic on $\Omega$ and $b$ is some boundary operator such that the problem (1.1), (1.2) has a unique solution.

Suppose that for each $0<h<1$ we are given a linear space of finite elements $S_{h}$ (which we roughly think of as being a space of piecewise polynomials defined on some partition of $\Omega$ ) and an approximate solution $u_{h} \in S_{h}$ of (1.1), (1.2) determined by one of the many Ritz-Galerkin methods which have been proposed. For a survey

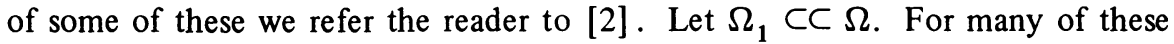
methods, $u_{h}$ satisfies the interior equations

$$
\begin{aligned}
B\left(u_{h}, \varphi\right) & =\int_{\Omega}\left(\sum_{i, j=1}^{N} a_{i j} \frac{\partial u_{h}}{\partial x_{i}} \frac{\partial \varphi}{\partial x_{j}}+\sum_{i=1}^{N} b_{i} \frac{\partial u_{n}}{\partial x_{i}} \varphi+c u_{h} \varphi\right) d x \\
& =B(u, \varphi)=\int_{\Omega} f \varphi d x
\end{aligned}
$$

for all $\varphi \in \stackrel{\circ}{S}_{h}\left(\Omega_{1}\right)$, where $\stackrel{\circ}{S}_{h}\left(\Omega_{1}\right)=\left\{\varphi \mid \varphi \in S_{h}(\Omega)\right.$, $\left.\operatorname{supp}(\varphi) \subset \Omega_{1}\right\}$. Roughly speaking, suppose that $S_{h}$ has the property (and some others described in Section 3 ) that for each $u$ belonging to the Sobolev space $H^{r}$,

$$
\inf _{\psi \in S_{h}}\|u-\psi\|_{1, \Omega_{1}} \leqslant C h^{r-1}\|u\|_{r, \Omega_{1}} .
$$

Received July 15, 1976.

AMS (MOS) subject classifications (1970). Primary 65N30.

* This work was supported in part by a grant from the National Science Foundation. 
Here $r \geqslant 2$ is a given integer and for $s \geqslant 0,\|\cdot\|_{s, \Omega_{1}}$ denotes the norm on $H^{s}\left(\Omega_{1}\right)$. It was shown in [15] that for $\Omega_{0} \subset \subset \Omega_{1}$ the interior estimate

$$
\left\|u-u_{h}\right\|_{0, \Omega_{0}} \leqslant C\left\{h^{r}\|u\|_{r, \Omega_{1}}+\left\|u-u_{h}\right\|_{-p, \Omega_{1}}\right\}
$$

holds. Here $p$ is a nonnegative integer and $\|\cdot\|_{-p, \Omega_{1}}$ is the norm on the dual space of $\stackrel{\circ}{H}^{p}\left(\Omega_{1}\right)$ (the completion of $C_{0}^{\infty}\left(\Omega_{1}\right)$ under $\left.\|\cdot\|_{p}\right)$. Under the further assumption that the restriction of elements of $S_{h}$ to $\Omega_{1}$ are piecewise polynomials defined on a uniform mesh, it was shown in [4] that

$$
\left|u-u_{h}\right|_{0, \Omega_{0}} \leqslant C\left\{h^{r}\|u\|_{r+[N / 2]+1, \Omega_{1}}+\left\|u-u_{h}\right\|_{-p, \Omega_{1}}\right\} .
$$

Here $|\cdot|_{0, \Omega_{0}}$ denotes the maximum norm on $\bar{\Omega}_{0}$ and $[N / 2]$ is the integral part of $N / 2$.

For many methods, estimates for $\|e\|_{-p, \Omega_{1}}$ already exist in the literature. Noting that $\|e\|_{-p, \Omega} \leqslant\|e\|_{-p, \Omega}$ and taking $p=r-2$, one often finds that $\left\|u-u_{h}\right\|_{2-r, \Omega} \leqslant C h^{2 r-2}\|u\|_{r, \Omega}$. Hence in these cases, if $u \in H^{r}(\Omega)$ and $r \geqslant 3$, this latter term is of higher order in $h$ than the first terms on the right in (1.4) and (1.5).

Instead of considering $u_{h}$, in this paper we shall consider certain "averages" of $u_{h}$ as approximations to $u$. More precisely, the averages are formed by computing $K_{h} * u_{h}$, where $K_{h}$ is a fixed function and $*$ denotes convolution. We shall see that the function $K_{h}$ has the following properties:

(i) $K_{h}$ has small support,

(ii) $K_{h}$ is independent of the specific choice of $S_{h}$ or the operator $L$,

(iii) $K_{h} * u_{h}$ is easily computable from $u_{h}$.

Furthermore we shall prove the following estimates (where again we assume $S_{h}$ to be defined on a uniform mesh on $\Omega_{1}$ ):

$$
\left\|u-K_{h} * u_{h}\right\|_{0, \Omega_{0}} \leqslant C\left\{h^{2 r-2}\|u\|_{2 r-2, \Omega_{1}}+\left\|u-u_{h}\right\|_{-p, \Omega_{1}}\right\}
$$

and

$$
\left|u-K_{h} * u_{h}\right|_{0, \Omega_{0}} \leqslant C\left\{h^{2 r-2}\|u\|_{2 r-2+[N / 2]+1, \Omega_{1}}+\left\|u-u_{h}\right\|_{-p, \Omega_{1}}\right\} .
$$

Hence in the cases in which $r \geqslant 3$ and $\left\|u-u_{h}\right\|_{2-r, \Omega_{1}} \leqslant C h^{2 r-2}\|u\|_{r, \Omega}, K_{h} * u$ is of order $h^{2 r-2}$ locally and therefore, for $h$ sufficiently small, better approximates $u$ than does $u_{h}$.

It is important to note that $K_{h} * u_{h}$ may be computed at each point in $\Omega_{0}$. However if we restrict our attention to specific points, say, for example, mesh points, then it very often takes on a very simple form; this will be discussed in Section 5.

An announcement of some of the results obtained in this paper was given in [7] For some superconvergence type results for two point boundary value problems the reader is referred to [9], [10] and [11]. For some interior superconvergence type results for multi-dimensional elliptic problems see [6]. For parabolic problems we refer the reader to [17] and [18].

An outline of this paper is as follows: In Section 2 we collect some notation. 
In Section 3 we discuss the necessary properties of the subspaces and in Section 4 we discuss the interior equations and collect some further preliminaries. In Section 5 the averaging operator is defined and its computation is discussed. Section 6 contains our main superconvergence results. In Section 7 we give some applications of the results and in one example show how to obtain superconvergence up to the boundary when $\Omega$ is the unit square, $L u=-\Delta u$ in $\Omega$ and $b u \equiv u$ on $\partial \Omega$.

2. Notation and Some Preliminaries. Let $\Omega$ be a bounded open set in $\mathbf{R}^{N}$. For $m$ a nonnegative integer, $C^{m}(\Omega)$ will denote the space of real-valued functions having continuous derivatives up to order $m$ on $\bar{\Omega}$ with the norm

$$
|u|_{m}=\sum_{|\alpha| \leqslant m} \sup _{x \in \bar{\Omega}}\left|D^{\alpha} u(x)\right|
$$

$C_{0}^{\infty}(\Omega)$ will denote the infinitely differentiable functions on $\Omega$ whose support is contained in $\Omega . H^{m}(\Omega)\left(\operatorname{resp} . \stackrel{\circ}{H}^{m}(\Omega)\right)$ is the completion of $C^{\infty}(\Omega)\left(\right.$ resp. $\left.C_{0}^{\infty}(\Omega)\right)$ with respect to the norm

$$
\|u\|_{m, \Omega}=\left(\sum_{|\alpha| \leqslant m} \int_{\Omega}\left|D^{\alpha} u\right|^{2} d x\right)^{1 / 2} .
$$

Note that $H^{0}(\Omega)=\stackrel{\circ}{H}^{0}(\Omega)=L_{2}(\Omega)$.

For $m$ a nonnegative integer $H^{-m}(\Omega)$ is the completion of $C_{0}^{\infty}(\Omega)$ with respect to the norm

$$
\|u\|_{-m, \Omega}=\sup _{v \in C_{0}^{\infty}(\Omega)} \frac{\int_{\Omega} u v d x}{\|v\|_{m, \Omega}}=\sup _{v \in C_{0}^{\infty}(\Omega)} \frac{(u, v)}{\|v\|_{m, \Omega}} .
$$

For $\beta$ a multi-integer define the translation operator $T_{h}^{\beta} u(x)=u(x+\beta h)$ and the difference quotients,

$$
\partial_{h, j} u=h^{-1}\left(T_{h / 2}^{e_{j}}-T_{h / 2}^{-e_{j}}\right) u
$$

Here $e_{j}$ is the multi-index whose $j$ th component is 1 and all others 0 . For any multiindex $\alpha$ we set

$$
\partial_{h}^{\alpha} u=\left(\partial_{h, 1}^{\alpha} \cdots \partial_{h, N}^{\alpha}\right) u
$$

We shall also need the following results:

LEMмA 2.1 (cf. e.g. [12]). Let $\Omega_{0} \subset \subset \Omega_{1}$. If $u \in H^{[N / 2]+1}\left(\Omega_{1}\right)$, then (after possible modification on a set of measure zero) $u \in C^{0}\left(\Omega_{0}\right)$ and

$$
|u|_{0, \Omega_{0}} \leqslant C\|u\|_{[N / 2]+1, \Omega_{0}}
$$

where $C=C\left(\Omega_{0}, \Omega_{1}\right)$ and $[N / 2]$ is the integral part of $N / 2$.

L EMMA 4.2. Let $\Omega_{0} \subset \subset \Omega_{1}$ and $s$ be an arbitrary but fixed nonnegative integer. Then there is a constant $C$ such that

$$
\|u\|_{0, \Omega_{0}} \leqslant C \sum_{|\alpha| \leqslant s}\left\|D^{\alpha} u\right\|_{-s, \Omega_{1}} .
$$


Proof. Without loss of generality we may assume that $\Omega_{1}$ is smooth and $\Omega_{0} \subset \subset$ $\Omega_{1} \subset \subset \mathbf{R}^{N}$. First suppose that supp $u \subset \subset \Omega_{1}$. In this case the proof proceeds by induction. For $s=0(2.5)$ is trivial. Assume (2.5) for $s \geqslant 0$, then for any $\alpha$ with $|\alpha| \leqslant s$

$$
\left\|D^{\alpha} u\right\|_{-s, \Omega_{1}}=\sup _{v \in C_{0}^{\infty}\left(\Omega_{1}\right)} \frac{\left(D^{\alpha} u, v\right)}{\|v\|_{s, \Omega_{1}}}
$$

Let $g$ be a solution of $-\Delta g=v$ in $\Omega_{1}, v=0$ on $\partial \Omega_{1}$. Since $u$ has compact support, integration by parts and Schwarz's inequality yields

$$
\begin{aligned}
\left(D^{\alpha} u, v\right) & =\sum_{i=1}^{N}\left(\frac{\partial}{\partial x_{i}} D^{\alpha} u, \frac{\partial g}{\partial x_{i}}\right) \\
& \leqslant C\left(\sum_{|\beta|=|\alpha|+1}\left\|D^{\beta} u\right\|_{-s-1, \Omega_{1}}\right)\|g\|_{s+2} .
\end{aligned}
$$

But by elliptic regularity (cf. [13])

$$
\|g\|_{s+2, \Omega_{1}} \leqslant C\|v\|_{s, \Omega_{1}}
$$

In view of this, (2.7) and (2.6), it follows that

$$
\sum_{|\alpha| \leqslant s}\left\|D^{\alpha} u\right\|_{-s, \Omega_{1}} \leqslant \sum_{|\beta| \leqslant s+1}\left\|D^{\beta} u\right\|_{-s-1, \Omega_{1}},
$$

which completes the proof for $u$ with compact support in $\Omega_{1}$. More generally let $w \in C^{\infty}\left(\Omega_{1}\right)$ with $w=1$ on $\Omega_{0}$ and supp $w \subset \subset \Omega_{1}$. Applying (2.5) to the function $w u$ we obtain

$$
\|u\|_{0, \Omega_{0}} \leqslant C \sum_{|\alpha| \leqslant s}\left\|D^{\alpha}(w u)\right\|_{-s, \Omega_{1}}
$$

Using Leibniz's rule and the definition of the $H^{-s}\left(\Omega_{1}\right)$ norm it is easily seen that

$$
\sum_{|\alpha| \leqslant s}\left\|D^{\alpha}(w u)\right\|_{-s, \Omega_{1}} \leqslant C \sum_{|\alpha| \leqslant s}\left\|D^{\alpha} u\right\|_{-s, \Omega_{1}},
$$

which completes the proof.

3. Piecewise Polynomial Subspaces. In the following sections we shall be concerned with Ritz-Galerkin methods in which the functions used for approximation are piecewise polynomials on a uniform mesh. More precisely, let $\left\{Q_{\beta}\right\}, \beta \in Z^{N}$ (the multi-integers) denote a family of disjoint open sets which partition $\mathbf{R}^{N}$. We shall assume that the partition is invariant under translations by $\nu$, for any $\nu \in Z^{N}$. Let $r \geqslant 2$ be an integer. $S_{1}^{r}$ will denote a linear space of functions which are polynomials on each $Q_{\beta}$ and belong to $H^{1}(\Omega)$ for any $\Omega \subset \subset \mathbf{R}^{N}$. Furthermore we assume that $S_{1}^{r}$ is translation invariant in the sense that if $\varphi \in S_{1}^{r}$, then $T_{1}^{\alpha} \varphi \in S_{1}^{r}$, for all $\alpha \in Z^{N}$.

For each $0<h \leqslant 1$, let $Q_{\beta}^{h}=h Q_{\beta}$. Then the family $\left\{Q_{\beta}^{h}\right\}$ forms a partition of $\mathbf{R}^{N}$ which is invariant under translations by $h \nu, \nu \in Z^{N}$. For $\Omega_{1} \subset \subset \mathbf{R}^{N}, S_{h}^{r}\left(\Omega_{1}\right)$ will denote the finite-dimensional subspace of $H^{1}\left(\Omega_{1}\right)$ consisting of functions which are the restrictions to $\Omega_{1}$ of functions of the form $\varphi(x / h)$, where $\varphi(x) \in S_{1}^{r}$. Let $\stackrel{\circ}{S}_{h}^{r}(\Omega)=$ 
$\left\{\varphi \mid \varphi \in S_{h}^{r}(\Omega)\right.$, supp $\left.\varphi \subseteq \Omega\right\}$. We note that if $\Omega_{0} \subset \subset \Omega_{1}$ and $\alpha \in Z^{N}$, then for $h$ sufficiently small (depending on $\alpha$ ), $\partial_{h}^{\alpha} \varphi \in \stackrel{\circ}{S}_{h}^{r}\left(\Omega_{1}\right)$ for all $\varphi \in \stackrel{\circ}{S}_{h}^{r}\left(\Omega_{0}\right)$. We shall also make the following approximability assumptions: $u \in \stackrel{\circ}{H}^{j}\left(\Omega_{0}\right)$

A.1. Let $\Omega_{0} \subset \subset \Omega_{1}$. There exists an $h_{0}>0$ such that for all $h \in\left(0, h_{0}\right]$ and

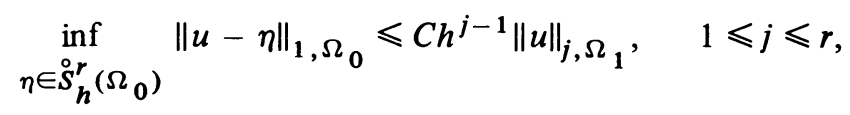

where $C$ is independent of $u$ and $h$.

A.2. If $\varphi \in S_{h}^{r}\left(\Omega_{1}\right), h \in\left(0, h_{0}\right], \Omega_{0} \subset \subset \Omega_{1}, \omega \in C_{0}^{\infty}\left(\Omega_{0}\right)$, then there exists an $\eta \in \stackrel{\circ}{S}_{h}^{r}\left(\Omega_{1}\right)$ such that

$$
\|\omega \varphi-\eta\|_{1, \Omega_{0}} \leqslant C h\|\varphi\|_{1, \Omega_{1}}
$$

where $C$ is independent of $h$ and $\varphi$.

Often in practice subspaces which satisfy the above conditions can be described in the following manner: There exist functions $\varphi_{1}, \ldots, \varphi_{m}$, in $H^{1}$, which are piecewise polynomials with compact support, such that $\varphi \in S_{h}^{r}(\Omega)$ is of the form

$$
\varphi(x)=\sum_{j=1}^{m} \sum_{\beta \in Z^{N}} a_{\beta}^{j} \varphi_{j}\left(h^{-1} x-\beta\right) \quad \text { for } x \in \Omega .
$$

Here the coefficients $a_{\beta}^{j}$ are real.

Example 1. An example of subspaces of this type are those generated by the $B$-splines of Schoenberg [16]. These will play a central role in what follows. Let us describe them more precisely.

For $t$ real, define

$$
\chi(t)= \begin{cases}1, & |t| \leqslant 1 / 2 \\ 0, & |t|>1 / 2\end{cases}
$$

and for $l$ an integer, set

$$
\psi_{1}^{(l)}(t)=\chi * \chi * \cdots * \chi, \quad \text { convolution } l-1 \text { times. }
$$

The function $\psi_{1}^{(l)}$ is the one-dimensional $B$-spline basis function of order $l$. Then

$$
\psi^{(l)}(x)=\prod_{j=1}^{N} \psi_{1}^{(l)}\left(x_{j}\right)
$$

is the $N$-dimensional $B$-spline basis function of order $l$. In this case every element of $S_{h}$ on $\Omega$ is of the form

$$
\varphi(x)=\sum_{\alpha \in Z^{N}} a_{\alpha} \psi^{(l)}\left(h^{-1} x-\alpha\right), \quad x \in \Omega .
$$

Example 2. A more general class of splines than (3.6) which satisfy our assumptions are the tensor products of one-dimensional splines. They may be described as follows: Let us subdivide $\mathbf{R}^{1}$ into intervals $I_{j}$ of length $h$. Let $M_{k, r}^{h}\left(\mathbf{R}^{1}\right)=\{\varphi \mid \varphi \in$ $P_{r-1}\left(I_{j}\right), \varphi \in C^{k}(\Omega)$ for any $\left.\Omega \subset \subset \mathbf{R}^{1}\right\}$, where $P_{r-1}\left(I_{j}\right)$ are the polynomials of degree 
$\leqslant r-1$ restricted to $I_{j} . M_{k, r}^{h}\left(\mathbf{R}^{N}\right)$ is then defined to be the $N$-fold tensor product of $M_{k, r}^{h}\left(\mathbf{R}^{1}\right)$ and for any $\Omega \subset \subset \mathbf{R}^{N}, M_{k, r}^{h}(\Omega)$ is the restriction of $M_{k, r}^{h}\left(\mathbf{R}^{N}\right)$ to $\Omega$. We note that if $k=r-2$ then these are the $B$-splines defined above. If $m$ is a positive integer, $r=2 m$ and $k=m-1$, then these are the so-called piecewise Hermite polynomials (cf. [4]).

Example 3. It was shown in [15] that the triangular elements of Bramble and Zlámal [8] satisfy our conditions. Here we take them on a uniform triangulation of $\mathbf{R}^{N}$.

4. Interior Equations for Ritz-Galerkin Methods and Some Preliminaries. Let $\Omega_{1}$ be a bounded open set in $\mathbf{R}^{N}$ and $B(u, v)$ a bilinear form defined on $H^{1}\left(\Omega_{1}\right) \times H^{1}\left(\Omega_{1}\right)$ given by

$$
B(u, v)=\int_{\Omega_{1}}\left(\sum_{i, j=1}^{N} a_{i j}(x) D_{i} u D_{j} v+\sum_{i=1}^{N} b_{i}(x)\left(D_{i} u\right) v+c(x) u v\right) d x
$$

where for simplicity the coefficients $a_{i j}, b_{i}$, are assumed to be of class $C^{\infty}\left(\Omega_{1}\right)$ and $a_{i j}$ $=a_{j i}$. We note that in general $B(u, v)$ may not be symmetric. We assume throughout that $B(u, v)$ is uniformly elliptic on $\Omega_{1}$; i.e., there exists a constant $C>0$ such that for all $x \in \bar{\Omega}_{1}$ and real vectors $\xi=\left(\xi_{1}, \ldots, \xi_{N}\right) \neq 0$,

$$
C \sum_{i=1}^{N} \xi_{i}^{2} \leqslant \sum_{i, j=N}^{N} a_{i j} \xi_{i} \xi_{j}
$$

Let $u \in H^{1}\left(\Omega_{1}\right)$ and $u_{h} \in S_{h}^{r}\left(\Omega_{1}\right)$ and suppose that $u-u_{h}$ satisfies the "interior" Ritz-Galerkin equations

$$
B\left(u-u_{h}, \varphi\right)=0
$$

for all $\varphi \in \stackrel{\circ}{S}_{h}^{r}\left(\Omega_{1}\right)$. Here $u_{h}$ may be thought of as an approximation to $u$ on $\Omega_{1}$ obtained by using some Ritz-Galerkin method on a larger set $\Omega$. Instead of investigating the properties of the error $u-u_{h}$ we shall be interested in investigating the properties of the difference between $u$ and certain averages of $u_{h}$. In order to do so we shall need some results concerning $u-u_{h}$ derived in [15] and [4] .

LEMмA 4.1. Let $\Omega_{0} \subset \subset \Omega_{1} \subset \subset \mathbf{R}^{N}, u \in H^{r+|\alpha|}\left(\Omega_{1}\right), \alpha$ a multi-index, $u_{h} \in$ $S_{r}^{h}\left(\Omega_{1}\right), r \geqslant 2$ and $p$ be a nonnegative integer, arbitrary but fixed. Suppose that A.1 and A.2 are satisfied and that $u-u_{h}$ satisfies (4.2). Then for $h$ sufficiently small

$$
\left\|\partial_{h}^{\alpha}\left(u-u_{h}\right)\right\|_{2-r, \Omega_{0}} \leqslant C\left(h^{2 r-2}\|u\|_{r+|\alpha|, \Omega_{1}}+\left\|u-u_{h}\right\|_{-p, \Omega_{1}}\right) .
$$

Here $C$ is independent of $u, u_{h}$ and $h$ but in general depends on $p, \alpha, \Omega_{0}, \Omega_{1}$ and the coefficients $a_{i j}, b_{i}$ and $c$.

Proof. Clearly it is sufficient to prove (4.3) for $\partial_{h}^{\alpha}$ replaced by the forward difference operator $\widetilde{\partial}_{h}^{\alpha}=T_{h / 2}^{\alpha} \partial_{h}^{\alpha}$, and $\Omega_{0}$ replaced by $\Omega_{0}^{\prime}$ with $\Omega_{0} \subset \subset \Omega_{0}^{\prime}$. Now let $\Omega_{0} \subset \subset \Omega_{0}^{\prime} \subset \subset \Omega_{1}^{\prime} \subset \subset \Omega_{1}$. It follows from (6.8) of [15], that for $h$ sufficiently small and $e=u-u_{h}$ 


$$
\begin{aligned}
& B\left(\widetilde{\partial}_{h}^{\alpha} e, \varphi\right)=\int_{\Omega_{0}} \sum_{i, j=1}^{N} \sum_{\beta<\alpha}\left(\begin{array}{c}
\alpha \\
\alpha-\beta
\end{array}\right) \widetilde{\partial}_{h}^{\alpha-\beta} a_{i j} T_{h}^{\alpha-\beta} \widetilde{\partial}_{h}^{\beta} D_{i} e D_{j} \varphi d x, \\
& \forall \varphi \in \stackrel{\circ}{S}^{h}\left(\Omega_{0}^{\prime}\right) .
\end{aligned}
$$

Hence, referring to [15] in particular, from (5.6) of Theorem 5.2, (4.3) of Lemma 4.2, (6.5) following Theorem 6.1 and (4.4) above we have that

$$
\left\|\widetilde{\partial}_{h}^{\alpha} e\right\|_{2-r, \Omega_{0}^{\prime}} \leqslant C\left(h^{2 r-2}\|u\|_{r-2+|\alpha|, \Omega_{1}^{\prime}}+\|e\|_{-p, \Omega_{1}^{\prime}}+\sum_{\beta<\alpha}\left\|\widetilde{\partial}_{h}^{\beta} e\right\|_{2-r, \Omega_{1}^{\prime}}\right) \text {. }
$$

The proof now proceeds by induction on $|\alpha|$. If $\alpha=0$ then (4.3) follows from (4.5). Assume now that (4.3) holds for $|\alpha| \leqslant m$. If $|\alpha|=m+1$, then applying (4.3) to the third term on the right of (4.5) on the domains $\Omega_{1}^{\prime}$ and $\Omega_{1}$ the inequality (4.3) for $|\alpha|=m+1$ is obtained, which completes the proof.

LEMMA 4.2 [4]. Under the conditions of Lemma 4.1 there exists a constant $C>0$ such that

$$
\left|\partial_{h}^{\alpha}\left(u-u_{h}\right)\right|_{0, \Omega_{0}} \leqslant C\left(h^{r}\|u\|_{r+|\alpha|+[N / 2]+1, \Omega_{1}}+\left\|u-u_{h}\right\|_{-p, \Omega_{1}}\right),
$$

where $C$ is independent of $u, u_{h}$ and $h$ but in general depends on $p, \alpha, \Omega_{0}, \Omega_{1}$ and the coefficients $a_{i j}, b_{i}$ and $c$.

5. A Class of Convolution Operators. In this section we shall introduce a particular class of convolution operators and discuss some of their properties. In the next section we shall use these to show (under certain conditions) that a certain convolution of the Ritz-Galerkin solution $u_{h}$ is closer to $u$ than $u_{h}$ is itself.

We shall begin by defining the kernels of the above-mentioned convolution operators. In each case it is an $N$-dimensional smooth spline which is the product of a single suitably chosen one-dimensional smooth spline. More precisely let $\psi_{1}^{(l)}$ be the one-dimensional smooth spline of order $l$ defined by (3.4). For $l=r-2, r \geqslant 2$ given, and $x \in \mathbf{R}^{N}$ set

$$
K_{h}(x)=\prod_{m=1}^{N}\left(\sum_{j=-(r-2)}^{r-2} h^{-1} k_{j}^{\prime} \psi_{1}^{(r-2)}\left(h^{-1} x_{m}-j\right)\right)
$$

Here the constants $K_{j}^{\prime}$ are defined as follows:

(i) $k_{-j}^{\prime}=k_{j}^{\prime}, j=0, \ldots, r-2$.

(ii) $k_{0}^{\prime}=k_{0}$ and $k_{j}^{\prime}=k_{j / 2}, j=1, \ldots, r-2$,

where the $k_{j}, j=0, \ldots, r-2$, are determined as the unique solution (see Lemma 5.1 below) of the linear system of algebraic equations.

$$
\sum_{j=0}^{r-2} k_{j} \int_{R_{1}} \psi_{1}^{(r-2)}(y)(y+j)^{2 m} d y= \begin{cases}1 & \text { if } m=0, \\ 0 & \text { if } m=1, \ldots, r-2 .\end{cases}
$$

We note that the constants $k_{j}$ can be easily computed and depend only on the choice of $r$.

The convolution operators in which we are interested are of the form 


$$
\left(K_{h} * u\right)(x)=h^{-N} \int_{\mathbf{R}^{N}}\left[\prod_{m=1}^{N} \sum_{j=2-r}^{r-2} k_{j}^{\prime} \psi_{1}^{(r-2)}\left(h^{-1}\left(x_{m}-y_{m}\right)-j\right)\right] u(y) d y
$$

If we set $k_{j}^{\prime}=0$ for $|j| \geqslant r-1$ and for $\beta$ a multi-integer define $k_{\beta}^{\prime}=\Pi_{j=1}^{N} k_{\beta_{j}}^{\prime}$, then (5.2) and (5.3) may be rewritten as

$$
K_{h}(x)=h^{-N} \sum_{\beta} k_{\beta}^{\prime} \psi^{(r-2)}\left(h^{-1} x-\beta\right)
$$

and

$$
\left(K_{h} * u\right)(x)=h^{-N} \int_{\mathbf{R}^{N}} \sum_{\beta} k_{\beta}^{\prime} \psi^{(r-2)}\left(h^{-1}(x-y)-\beta\right) u(y) d y,
$$

respectively.

In what follows we shall be interested in $K_{h} * u_{h}$ as an approximation to $u$. Before investigating the properties of $K_{h}$, let us first consider the computation of $K_{h} *$ $u_{h}$. If, in the region to be studied, $u_{h}$ is of the form (3.3), that is

$$
u_{h}=\sum_{j=1}^{K} \sum_{\alpha \in Z^{N}} a_{\alpha}^{j} \varphi_{j}\left(h^{-1} x-\alpha\right)
$$

then using (5.5) and making a change in variables we have

$$
\begin{aligned}
& \left(K_{h} * u_{h}\right)(x) \\
& \quad=\sum_{j=1}^{m} \sum_{\alpha \in Z^{N}} a_{\alpha}^{j}\left(\sum_{\beta \in Z^{N}} k_{\beta}^{\prime} \int_{\mathbf{R}^{N}} \psi^{r-2}\left(h^{-1} x-\eta-\beta-\alpha\right) \varphi_{j}(\eta) d x\right) .
\end{aligned}
$$

Although the terms $k_{\beta}^{\prime} \int_{\mathbf{R}^{N}} \psi^{l}\left(h^{-1} x-\eta-\beta-\alpha\right) \varphi_{j}(\eta) d \eta$ and hence $K_{h} * u_{h}$ may be calculated at any point, we shall, for simplicity restrict our attention to points of the form $x=h \gamma, \gamma \in Z^{N}$. In this case

$$
\left(K_{h} * u_{h}\right)(h \gamma)=\sum_{j=1}^{m} \sum_{\alpha \in Z^{N}} a_{\alpha}^{j}\left(\sum_{\beta \in Z^{N}} k_{\beta}^{\prime} \int_{\mathbf{R}^{N}} \psi^{r-2}(\gamma-\beta-\alpha-\eta) \varphi_{j}(\eta) d \eta\right)
$$

or

$$
\left(K_{h} * u_{h}\right)(h \gamma)=\sum_{j=1}^{m} \sum_{\gamma, \delta \in Z^{N}} a_{\gamma-\delta}^{j} d_{\delta}^{j}
$$

where

$$
d_{\delta}^{j}=\sum_{\beta \in Z^{N}} k_{\beta}^{\prime} \int_{\mathbf{R}^{N}} \psi^{r-2}(\delta-\beta-\eta) \varphi_{j}(\eta) d \eta
$$

Thus the values of $K_{h} * u_{h}$ at mesh points are determined by taking a fixed finite linear combination of the values of the coefficients $a_{\alpha}^{j}$. There are only a finite number of nonzero coefficients $d_{\delta}^{j}$. They may be computed a priori and are independent of $h$ and the particular mesh point.

As an example, let us take the case where $u_{h}$ itself is a smooth spline. Then 
$k=1, \varphi_{1}=\psi^{(r)}$ and $u_{h}$ is of the form

$$
u_{h}=\sum_{\alpha \in Z^{N}} a_{\alpha} \psi^{(r)}\left(h^{-1} x-\alpha\right)
$$

Setting $d_{\delta}^{j}=d_{\delta}$ in this case, we have

$$
d_{\delta}=\sum_{\beta \in Z^{N}} k_{\beta}^{\prime} \psi^{2 r-2}(\delta-\beta)=\prod_{l=1}^{N} d_{\delta_{l}}^{\prime} \equiv \prod_{l=1}^{N}\left(\sum_{\eta=-(t-1)}^{t-1} k_{\eta}^{\prime} \psi_{1}^{2 r-2}\left(\delta_{l}-\eta\right)\right) .
$$

Here the $d_{l}^{\prime}$ correspond to the weights $d_{\delta}$ in the one-dimensional case. A table of the one-dimensional coefficients $d_{l}^{\prime}$ from which the $N$-dimensional weights $d_{\delta}$ in (5.10) may be formed will be given in the appendix. We remark that the computation of the $d_{\delta}^{j}$ may also be reduced to the product of the one-dimensional case if we take $S_{h}$ to be the more general class of tensor products of one-dimensional splines given in Example 2 of Section 3.

For technical reasons in what follows we shall make use of a slightly more general class of convolution operators than those defined by (5.3). We shall now introduce these and discuss some of their properties. The following result will be needed.

Lemm A 5.1 (cf. [6, Lemma 8.1]). Let $t \geqslant 1$ and $l \geqslant 1$ be arbitrary but fixed integers. There exist uniquely determined real constants $k_{j}, j=0, \ldots, t-1$, which satisfy the linear system of algebraic equations.

$$
\sum_{j=0}^{t-1} k_{j} \int_{\mathrm{R}^{N}} \psi_{1}^{(l)}(y)(y+j)^{2 m} d y= \begin{cases}1 & \text { if } m=0 \\ 0 & \text { if } m=1, \ldots, t-1 .\end{cases}
$$

Here, with a slight abuse of notation, we have suppressed the dependence of the solutions of (5.11) on $t$ and $l$. We note that (5.2) and (5.11) coincide in the case $l=$ $r-2$ and $t=r-1$.

As before let $k_{0}^{\prime}=k_{0}, k_{j}^{\prime}=k_{j} / 2, k_{-j}^{\prime}=k_{j}^{\prime}$ for $j=1, \ldots, t-1$ and $k_{j}=0$ for $|j| \geqslant t$. For $\beta \in Z^{N}$ set $k_{\beta}^{\prime}=\Pi_{j=1}^{N} k_{\beta_{j}}^{\prime}$ and define

$$
\begin{aligned}
K_{h, l}^{2 t}(x) & =h^{-N} \sum_{\beta \in Z^{N}} k_{\beta}^{\prime} \psi^{l}\left(h^{-1} x-\beta\right) \\
& =\prod_{m=1}^{N}\left[h^{-1} \sum_{j=-(t-1)}^{t-1} k_{j}^{\prime} \psi_{1}^{(l)}\left(h^{-1} x_{m}-j\right)\right] .
\end{aligned}
$$

We remark that $\operatorname{supp}\left(K_{h, l}^{2 t}(x)\right)$ is the cube with side of length $(2 t+l) h$ centered at the origin. Note also that $K_{h, r-2}^{2 r-2}(x)=K_{h}(x)$.

The function $K_{h, l}(x)$ was constructed in such a way that $K_{h, l}^{2 t} * u$, where $*$ denotes convolution, is an approximation of order $h^{2 t}$ to $u$. More precisely we have the following:

Lемма 5.2. Let $t \geqslant 1$ and $l \geqslant 1$ be fixed integers and $\Omega_{0} \subset \subset \Omega_{1}$. Then for $h$ sufficiently small

$$
\left|u-K_{h, l}^{2 t} * u\right|_{0, \Omega_{0}} \leqslant C h^{s}|u|_{s, \Omega_{1}}, \quad 0 \leqslant s \leqslant 2 t,
$$




$$
\left\|u-K_{h, l}^{2 t} * u\right\|_{0, \Omega_{0}} \leqslant C h^{s}\|u\|_{s, \Omega_{1}}, \quad 0 \leqslant s \leqslant 2 t .
$$

The proofs will not be given. We only remark that (5.13) and (5.14) follow as a simple consequence of the Bramble-Hilbert Lemma (cf. [3]), using the fact that Lemma 5.1 implies that $K_{h, l}^{2 t} * u$ reproduces polynomials of degree not exceeding $2 t-1$ in each variable (cf. [6, Lemma 8.1]). We also note that (5.13) may also be proved using this latter fact and Taylor's Theorem.

We shall need some other properties of $K_{h, l}^{2 t} * u$.

LemmA 5.3. Let $t, l, h, \Omega_{0}$ and $\Omega_{1}$ be as above. Then

(i) For any multi-index $\alpha$ and $u \in L_{2}\left(\Omega_{1}\right)$

$$
\partial_{h}^{\alpha}\left(K_{h, l}^{2 t} * u\right)(x)=K_{h, l}^{2 t} * \partial_{h}^{\alpha} u(x), \quad x \in \Omega_{0} .
$$

(ii) If $s$ is any fixed integer (positive or negative) and $\alpha$ is any multi-index with $\alpha_{j} \leqslant l, j=1, \ldots, N$,

$$
\left\|D^{\alpha}\left(K_{h, l}^{2 t} * u\right)\right\|_{s, \Omega_{0}} \leqslant C\left\|\partial_{h}^{\alpha} u\right\|_{s, \Omega_{1}} \quad \text { for all } u \in H^{s}\left(\Omega_{1}\right)
$$

and

$$
\left|D^{\alpha}\left(K_{h, l}^{2 t} * u\right)\right|_{0, \Omega_{0}} \leqslant C\left|\partial_{h}^{\alpha} u\right|_{0, \Omega_{1}} \quad \text { for all } u \in C^{0}\left(\Omega_{1}\right)
$$

Here $C$ is a constant which is independent of $h$ and $u$.

Proof. The identity (5.15) follows very simply using a change of variables.

For simplicity in notation we shall prove (5.16) and (5.17) in the case $N=1$.

The multi-dimensional case follows in essentially the same manner. For $N=1$ we have

$$
\left(K_{h, l}^{2 t} * u\right)(x)=h^{-1} \int_{\mathrm{R}^{1}} \sum_{j=-(t-1)}^{t-1} k_{j}^{\prime} \psi_{1}^{(l)}\left(h^{-1}(x-y)-j\right) u(y) d y .
$$

If $0 \leqslant \alpha \leqslant l$ define

$$
V_{h, l-\alpha}^{2 t}(x)=h^{-1} \sum_{j=-(t-1)}^{t-1} k_{j}^{\prime} \psi_{1}^{(l-\alpha)}\left(h^{-1} x-j\right) .
$$

Then it is easy to see from (5.15) and (3.4) that

$$
D^{\alpha}\left(K_{h, l}^{2 t} * u\right)=V_{h, l-\alpha}^{2 t} * \partial_{h}^{\alpha} u .
$$

Now since $\psi_{1}^{(l-\alpha)} \geqslant 0, h^{-1} \int_{\mathbf{R}^{1}} \psi_{1}^{(l-\alpha)}\left(h^{-1} x-j\right) d x=1$ and $\operatorname{supp}\left(\psi_{1}^{(l-\alpha)}\left(h^{-1} x\right)\right)$ is an interval of length $(l+1-\alpha) h$, it follows that for $h$ sufficiently small

$$
\left|V_{h, l-\alpha}^{2 t} * \partial_{h}^{\alpha} u\right|_{0, \Omega_{0}} \leqslant C\left|\partial_{h}^{\alpha} u\right|_{0, \Omega_{1}}
$$

and

$$
\left\|V_{h, l-\alpha}^{2 t} * \partial_{h}^{\alpha} u\right\|_{0, \Omega_{0}} \leqslant C\left\|\partial_{h}^{\alpha} u\right\|_{0, \Omega_{1}} .
$$

The inequality (5.8) is just the inequality (5.17) and (5.19) is the inequality (5.16) in the case that $s=0$. In the case that $s$ is nonnegative, the inequality (5.16) follows from (5.9) on observing that for any nonnegative integer $m$, 


$$
D^{m}\left(D^{\alpha}\left(K_{h, l}^{2 t} * u\right)\right)=V_{h, l-2}^{2 t} * D^{m} \partial_{h}^{\alpha} u .
$$

For $s$ a negative integer we have

$$
\begin{aligned}
\left\|D^{\alpha} K_{h, l}^{2 t} * u\right\|_{s, \Omega_{0}} & =\sup _{v \in C_{0}^{\infty}\left(\Omega_{0}\right)} \frac{\left(D^{\alpha}\left(K_{h, l}^{2 t} * u\right), v\right)}{\|v\|_{-s, \Omega_{0}}} \\
& =\sup _{v \in C_{0}^{\infty}\left(\Omega_{0}\right)} \frac{\left(V_{h, l-\alpha}^{2 t} * \partial_{h}^{\alpha} u, v\right)}{\|v\|_{-s, \Omega_{0}}}=\sup _{v \in C_{0}^{\infty}\left(\Omega_{0}\right)} \frac{\left(\partial_{h}^{\alpha} u, V_{h, l-\alpha}^{2 t} * v\right)}{\|v\|_{-s, \Omega_{0}}} \\
& \leqslant \sup _{v \in C_{0}^{\infty}\left(\Omega_{0}\right)} \frac{\left\|\partial_{h}^{\alpha} u\right\|_{s, \Omega_{1}}\left\|V_{h, l-\alpha}^{2 t} * v\right\|_{-s, \Omega_{1}}}{\|v\|_{-s, \Omega_{0}}} .
\end{aligned}
$$

It is easily seen that

$$
\left\|v_{h, l-\alpha}^{2 t} * v\right\|_{-s, \Omega_{1}} \leqslant C\|v\|_{-s, \Omega_{0}}
$$

where $C$ is independent of $h$ and $v \in C_{0}^{\infty}\left(\Omega_{0}\right)$. This completes the proof.

6. Superconvergence Estimates. Let $u$ and $u_{h} \in S_{h}^{r}$ satisfy the interior equations (4.2). The inequalities (1.4) and (1.5) provide estimates for the error in the $L_{2}$ and maximum norm respectively. It is often the case that for specific problems the term $\|e\|_{-p, \Omega_{1}}$ is $O\left(h^{r}\right)$ and hence the estimates (1.4) and (1.5) yield $O\left(h^{r}\right)$ convergence locally. For many important problems $\|e\|_{-p, \Omega_{1}}$ is in fact $O\left(h^{2 r-2}\right)$. We shall show that if one considers $K_{h} * u_{h}$ instead of $u_{h}$ as an approximation to $u$, and if $\|e\|_{-p, \Omega_{1}}$ is $O\left(h^{2 r-2}\right)$, then $u-K_{h} * u_{h}$ is locally of order $h^{2 r-2}$ in both $L_{2}$ and maximum norms. Hence in these cases processing the solution $u_{h}$ yields higher order accuracy for $r \geqslant 3$.

We emphasize that $u-K_{h} * u_{h}$ will be $O\left(h^{2 r-2}\right)$ at every point of the region in which the error is measured. In practice one usually computes the solution at specific points, for example at mesh points. In this case $K_{h} * u_{h}$ takes a particularly simple form. This will also be discussed. We shall start with finding estimates for $u-K_{h} * u$ in $L_{2}$ norm (Theorem 1) and then in the maximum norm (Theorem 2).

THeOREM 1. Let $\Omega_{0} \subset \subset \Omega_{1}, u \in H^{2 r-2}\left(\Omega_{1}\right), u_{h} \in S_{h}^{r}\left(\Omega_{1}\right), r \geqslant 3$, where $S_{h}^{r}$ satisfies A.1 and A.2 and $u-u_{h}$ satisfies (4.2). Let $K_{h}$ be chosen as in (5.1) and let $p$ be an arbitrary but fixed nonnegative integer. Then for all $h$ sufficiently small

$$
\left\|u-K_{h} * u_{h}\right\|_{0, \Omega_{0}} \leqslant C\left(h^{2 r-2}\|u\|_{2 r-2, \Omega_{1}}+\left\|u-u_{h}\right\|_{-p, \Omega_{1}}\right),
$$

where $C$ is independent of $u$ and $h$.

Proof. By the triangle inequality

$$
\left\|u-K_{h} * u_{h}\right\|_{0, \Omega_{0}} \leqslant\left\|u-K_{h} * u\right\|_{0, \Omega_{0}}+\left\|K_{h} *\left(u-u_{h}\right)\right\|_{0, \Omega_{0}} .
$$

Let $\Omega_{0} \subset \subset \Omega_{0}^{\prime} \subset \subset \Omega_{0}^{\prime \prime} \subset \subset \Omega_{1}$. Since $K_{h}(x)=K_{h, r-2}^{2 r-2}(x)$ it follows from (5.14) that

$$
\left\|u-K_{h} * u\right\|_{0, \Omega_{0}} \leqslant C h^{2 r-2}\|u\|_{2 r-2, \Omega_{1}} .
$$


Now using (2.5), we have that

$$
\left\|K_{h} *\left(u-u_{h}\right)\right\|_{0, \Omega_{0}} \leqslant \sum_{|\beta| \leqslant r-2}\left\|D^{\beta} K_{h} *\left(u-u_{h}\right)\right\|_{2-r, \Omega_{0}^{\prime}} .
$$

The inequality (6.1) now follows from (5.16), (4.3) and (6.3).

In order to prove a maximum norm error estimate we shall make use of the following.

Lemma 6.1. Let $\Omega_{0} \subset \subset \Omega_{1}, v \in C^{0}(\Omega)$ and $N_{0}=[N / 2]+1$. Then for all $h$ sufficiently small

$$
\left|K_{h} * v\right|_{0, \Omega_{0}} \leqslant C\left[\sum_{|\alpha| \leqslant N_{0}+r-2}\left\|\partial_{h}^{\alpha} v\right\|_{2-r, \Omega_{1}}+h^{r-2} \sum_{|\alpha| \leqslant r-2}\left|\partial_{h}^{\alpha} v\right|_{0, \Omega_{1}}\right]
$$

where $C$ is independent of $v$ and $h$.

Proof. By the triangle inequality

$$
\left|K_{h} * v\right|_{0, \Omega_{0}} \leqslant\left|K_{h, N_{0}}^{2 r-2} * K_{h} * v\right|_{0, \Omega_{0}}+\left|K_{h} * v-K_{h, N_{0}}^{2 r-2} * K_{h} * v\right|_{0, \Omega_{0}}
$$

where $K_{h, N_{0}^{2}}^{2 r-2} * u$ is defined by (5.12). Let $\Omega_{0} \subset \subset \Omega_{0}^{\prime} \subset \subset \Omega_{1}$. Then using (2.4), (5.16), (5.15) and (2.5) we have for the first term on the right that

$$
\begin{aligned}
\left|K_{h, N_{0}}^{2 r-2} * K_{h} * v\right|_{0, \Omega_{0}} \leqslant C \sum_{|\alpha| \leqslant N_{0}}\left\|D^{\alpha} K_{h, N_{0}^{2 r-2}}^{2 r} K_{h} * v\right\|_{0, \Omega_{0}^{\prime}} \\
\quad \leqslant C\left(\sum_{|\alpha| \leqslant N_{0}}\left\|K_{h} * \partial_{h}^{\alpha} v\right\|_{0, \Omega_{0}^{\prime}}\right) \leqslant C\left(\sum_{|\alpha| \leqslant N_{0}+r-2}\left\|\partial_{h}^{\alpha} v\right\|_{2-r, \Omega_{1}}\right) .
\end{aligned}
$$

For the second term on the right of (6.6) we have

$$
\begin{aligned}
\left|K_{h} * v-K_{h, N_{0}}^{2 r-2} * K_{h} * v\right|_{0, \Omega_{0}} & \leqslant C h^{r-2}\left|K_{h} * v\right|_{r-2, \Omega_{0}^{\prime}} \\
& \leqslant C h^{r-2}\left(\sum_{|\alpha| \leqslant r-2}\left|\partial_{h}^{\alpha} v\right|_{0, \Omega^{\prime}}\right),
\end{aligned}
$$

where we have used (5.13) and (5.17). The inequality (6.5) now follows from (6.6), (6.7) and (6.8).

THEOREM 2. Suppose that the conditions of Theorem 1 are satisfied and that $u \in$ $H^{2 r-2+N_{0}}\left(N_{0}=[N / 2]+1\right)$. Then

$$
\left|u-K_{h} * u_{h}\right|_{0, \Omega_{0}} \leqslant C\left(h^{2 r-2}\|u\|_{2 r-2+N_{0}, \Omega_{1}}+\left\|u-u_{h}\right\|_{-p, \Omega_{1}}\right),
$$

where $C$ is independent of $u$ and $h$.

Proof. Using the triangle inequality and setting $u-u_{h}=e$,

$$
\left|u-K_{h} * u_{h}\right|_{0, \Omega_{0}} \leqslant\left|u-K_{h} * u\right|_{0, \Omega_{0}}+\left|K_{h} * e\right|_{0, \Omega_{0}} \text {. }
$$

Let $\Omega_{0} \subset \subset \Omega_{0}^{\prime} \subset \subset \Omega_{1}$. Then using (5.13) and (2.4) we have

$$
\left|u-K_{h} * u\right|_{0} \leqslant C h^{2 r-2}|u|_{2 r-2, \Omega_{0}^{\prime}} \leqslant C h^{2 r-2}\|u\|_{2 r-2+N_{0}, \Omega_{1}} .
$$

Applying Lemma 6.1, (4.3) and (4.4) 


$$
\begin{aligned}
\left|K_{h} * e\right|_{0, \Omega_{0}} & \leqslant C\left(\sum_{|\alpha| \leqslant r-2+N_{0}}\left\|\partial_{h}^{\alpha} e\right\|_{2-r, \Omega_{0}^{\prime}}+h^{r-2} \sum_{|\alpha| \leqslant r-2}\left|\partial_{h}^{\alpha} e\right|_{0, \Omega_{0}^{\prime}}\right) \\
& \leqslant C\left(h^{2 r-2}\|u\|_{2 r-2+N_{0}, \Omega_{1}}+\left\|u-u_{h}\right\|_{-p, \Omega_{1}}\right)
\end{aligned}
$$

which completes the proof.

Although $K_{h, r-2}^{2 r-2} * u_{h}$ can be calculated at arbitrary points in $\Omega_{0}$, Theorem 2 takes a particularly simple form if $u_{h}$ is of the form (3.3) and if, for example, we are interested in points in $\Omega_{0}$ of the form $h \gamma, \gamma \in Z^{N}$.

COROLLARY. Suppose the conditions of Theorem 2 are satisfied and $u_{h}$ is of the form (3.3). Then at points $h \gamma \in \bar{\Omega}_{0}, \gamma \in \mathbf{Z}^{N}$

$$
\begin{aligned}
& \sup _{h \gamma \in \bar{\Omega}_{0} ; \gamma \in \mathrm{Z}^{N}}\left|u(h \gamma)-\sum_{j=1}^{m} \sum_{\alpha} a_{\gamma-\alpha}^{j} d_{\alpha}^{j}\right| \\
& \leqslant C\left(h^{2 r-2}\|u\|_{2 r-2+N_{0}, \Omega_{1}}+\left\|u-u_{h}\right\|_{-p, \Omega_{1}}\right),
\end{aligned}
$$

where the $d_{\alpha}^{j}$ are given by (5.10).

7. Examples. Here the theory given in Section 6 will be exemplified, where for simplicity we shall restrict ourselves to discussing Dirichlet's problem. In Example 1 interior superconvergence estimates for two different methods will be discussed. In Example 2 a way of obtaining superconvergence up to the boundary, when the domain $\Omega$ is the unit square and the subspaces are taken to satisfy the boundary conditions, will be given.

Let $\Omega$ be a bounded domain in $\mathbf{R}^{N}$ with boundary $\partial \Omega$. For simplicity consider

$$
\begin{aligned}
-\Delta u=f & \text { in } \Omega, \\
u=0 & \text { on } \partial \Omega .
\end{aligned}
$$

For the purposes of the applications given here an additional assumption on the subspace $S_{h}^{r}(\Omega)$ will be made. Namely we suppose that there exists a constant $C$ independent of $u$ and $h$ such that for all $u \in H^{t}(\Omega) ; 1 \leqslant t \leqslant r$,

$$
\inf _{\chi \in S_{h}^{r}(\Omega)}\|u-\chi\|_{1, \Omega} \leqslant C h^{t-1}\|u\|_{t, \Omega} .
$$

In Example 2 the elements of $S_{r}^{h}(\Omega)$ are required to vanish on $\partial \Omega$. In this case (7.2) is assumed to hold only for $u \in \stackrel{\circ}{H}^{1}(\Omega) \cap H^{r}(\Omega)$.

In what follows we shall assume that the hypothesis of Theorems 1 and 2 are satisfied, where $\Omega_{0} \subset \subset \Omega_{1} \subset \subset \Omega$.

Example 1. Dirichlet's Problem on a Smooth Domain. Here we shall assume for simplicity that $\partial \Omega \in C^{\infty}$. In Babuška [1] and Nitsche [14], methods were introduced for approximating the solution of (7.1) in which the approximating subspaces need not satisfy the boundary condition. These methods have the same interior equations; i.e. if $u_{h}$ is the approximate solution determined by either of these methods, then 


$$
\begin{aligned}
\int_{\Omega} \sum_{i=1}^{N} \frac{\partial u_{h}}{\partial x_{i}} \frac{\partial \varphi}{\partial x_{i}} d x & =\int_{\Omega} f \varphi d x \\
& =\int_{\Omega} \sum_{i=1}^{N} \frac{\partial u}{\partial x_{i}} \frac{\partial \varphi}{\partial x_{i}} d x, \quad \forall \varphi \in \stackrel{\circ}{S}_{h}^{r}\left(\Omega_{1}\right) .
\end{aligned}
$$

What is important here is that we may choose $S_{h}^{r}(\Omega)$ to satisfy all of our conditions (cf. [4, Example 2], for more details). Now it was shown in [5] that the estimate

$$
\left\|u-u_{h}\right\|_{2-r, \Omega} \leqslant h^{r-1-t}\|u\|_{t, \Omega}
$$

is valid for $1 \leqslant t \leqslant r$. Using this inequality and the fact that $\left\|u-u_{h}\right\|_{2-r, \Omega_{1}} \leqslant$ $\left\|u-u_{h}\right\|_{2-r, \Omega}$, we obtain from Theorems 1 and 2 the error estimates

$$
\left\|u-K_{h} * u_{h}\right\|_{0, \Omega_{0}} \leqslant C h^{2 r-2}\left(\|u\|_{2 r-2, \Omega_{1}}+\|u\|_{r, \Omega}\right),
$$

and in the maximum norm

$$
\begin{aligned}
\mid u-K_{h} * & \left.u_{h}\right|_{0, \Omega_{0}} \\
& \leqslant C h^{2 r-2}\left(\|u\|_{2 r-2+[N / 2]+1, \Omega_{1}}+\|u\|_{r, \Omega}\right) .
\end{aligned}
$$

If we take $N=2$ and for example $S_{h}^{r}$ to be piecewise cubic Hermite polynomials or cubic smooth splines, then $r=4$ and (7.5) and (7.6) become

$$
\begin{gathered}
\left\|u-K_{h} * u_{h}\right\|_{0, \Omega_{0}} \leqslant C h^{6}\left(\|u\|_{6, \Omega_{1}}+\|u\|_{4, \Omega}\right), \\
\left|u-K_{h} * u_{h}\right|_{0, \Omega_{0}} \leqslant C h^{6}\left(\|u\|_{8, \Omega_{1}}+\|u\|_{4, \Omega}\right) .
\end{gathered}
$$

Example 2. Superconvergence Up to the Boundary for Dirichlet's Problem on a Square. Let $\Omega$ be the unit square in $\mathbf{R}^{2}$ (i.e. $\Omega=\left\{x \mid 0<x_{i}<1, i=1,2\right\}$ ), with boundary $\partial \Omega$ and let $u$ be the solution of (7.1).

Let $h=1 / n, n=1,2,3, \ldots$, and let $l$ be either 0 or 1 . We shall approximate $u$ taking our subspace $S_{h}^{r}(\Omega)=\bar{M}_{k, r}^{h}(\Omega)=\left\{\varphi\left|\varphi \in M_{l, r}^{h}(\Omega), \varphi\right|_{\partial \Omega}=0, k=0,1\right\}$. That is, $\bar{M}_{k, r}^{h}(\Omega)$ is the subspace of the space of tensor products of one-dimensional $C^{0}$ or $C^{1}$ splines (discussed in Section 3) consisting of elements which vanish on $\partial \Omega$.

Let $u_{h} \in S_{h}^{r}(\Omega)$ be the approximate solution of (7.1) defined by

$$
\begin{aligned}
\sum_{i=1}^{2} \int_{\Omega} \frac{\partial u_{h}}{\partial x_{i}} \frac{\partial \varphi}{\partial x_{i}} d x & =\int_{\Omega} f \varphi d x \\
& =\sum_{i=1}^{2} \int_{\Omega} \frac{\partial u}{\partial x_{i}} \frac{\partial \varphi}{\partial x_{i}} d x, \quad \forall S_{h}^{r}(\Omega) .
\end{aligned}
$$

We shall show that $u_{h}$ can be extended, in a simple manner, from $\Omega$ to a larger domain so that $u-K_{h} * u_{h}=O\left(h^{2 r-2}\right)$ on $\bar{\Omega}$ in both the $L_{2}$ and maximum norms. The extension $u_{h}$ can be described as follows: 


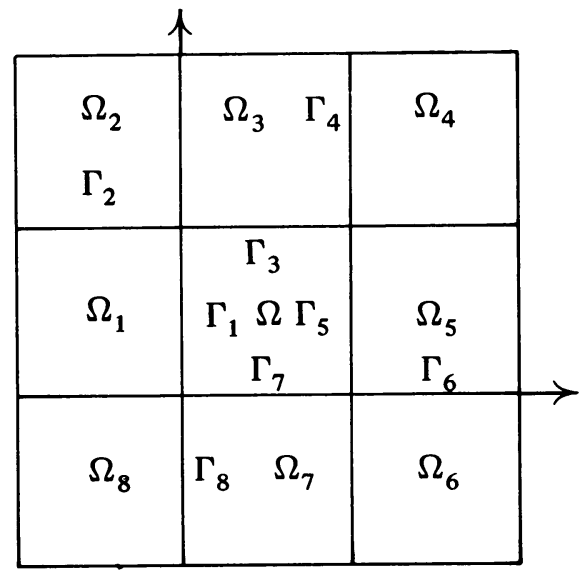

(i) Extend $u_{h}$ from $\Omega$ to $\Omega_{1}, \Omega_{3}, \Omega_{5}$ and $\Omega_{7}$ as an odd function by reflection across the faces $\Gamma_{1}, \Gamma_{3}, \Gamma_{5}$ and $\Gamma_{7}$ respectively.

(ii) Then extend $u_{h}$ to $\Omega_{2}, \Omega_{4}, \Omega_{6}$ and $\Omega_{8}$ as an odd function by reflection across the faces $\Gamma_{2}, \Gamma_{4}, \Gamma_{6}$ and $\Gamma_{8}$ respectively.

Notice that since $k$ is either 0 or $1, U_{h} \in \bar{M}_{k, r}^{h}\left(\Omega^{E}\right)$, where $\Omega^{E}=\left\{x \mid-1<x_{i}\right.$ $<2\}$.

THEOREM 3. Let $S_{h}^{r}$ and $U_{h}$ be defined as above with $r \geqslant 3$. If $h$ is sufficiently small and

(i) $f \in \stackrel{\circ}{H}^{2 r-4}(\Omega)$, then

$$
\left\|u-K_{h} * U_{h}\right\|_{0, \Omega} \leqslant C\left(h^{2 r-2}\|f\|_{2 r-4, \Omega}\right) .
$$

(ii) If $f \in \stackrel{\circ}{H}^{2 r-2}(\Omega)$, then

$$
\left|u-K_{h} * U_{h}\right|_{0, \Omega} \leqslant C\left(h^{2 r-2}\|f\|_{2 r-2, \Omega}\right),
$$

where $C$ is independent of $h$ and $f$.

Proof. Extend $u$ to $\Omega^{E}$ in the same manner as $U_{h}$ and call its extension $U$. Let $\Lambda_{i}, i=1,2,3,4$, be the squares

$$
\begin{array}{ll}
\Lambda_{1}=\left\{x \mid-1<x_{1}<1,0<x_{2}<2\right\}, & \Lambda_{2}=\left\{x \mid 0<x_{1}<2,0<x_{2}<2\right\}, \\
\Lambda_{3}=\left\{x \mid-1<x_{1}<1,-1<x_{2}<1\right\}, & \Lambda_{3}=\left\{x \mid 0<x_{1}<2,-1<x_{2}<1\right\} .
\end{array}
$$

We first claim that

$$
\sum_{j=1}^{2} \int_{\Lambda_{i}} \frac{\partial\left(U-U_{h}\right)}{\partial x_{j}} \frac{\partial \varphi}{\partial x_{j}} d x=0, \quad \forall \varphi \in \bar{M}_{k}^{h}\left(\Lambda_{i}\right), i=1,2,3,4
$$

Note that $U_{h} \in \bar{M}_{k}^{h}\left(\Lambda_{i}\right)$ and if $f \in \stackrel{\circ}{H}^{s}(\Omega)\left(s \geqslant 0\right.$ an integer) then $U \in H^{s+2}\left(\Lambda_{i}\right) \cap$ $\stackrel{\circ}{H}^{1}\left(\Lambda_{i}\right), i=1,2,3,4$. Furthermore

$$
\|U\|_{s+2, \Lambda_{i}} \leqslant C\|f\|_{s, \Omega}, \quad i=1,2,3,4 .
$$

Before proving (7.10), let us show how (7.8) and (7.9) follow from it. Equation (7.10) says that $U_{h}$ is the Ritz-Galerkin approximation in $\bar{M}_{k}^{h}\left(\Lambda_{i}\right)$ of $U$ on each of the 
domains $\Lambda_{i}, i=1,2,3,4$. Hence on each of these domains the interior estimates obtained in Theorems 1 and 2 are applicable. Let $\Omega_{0}^{i} \subset \subset \Lambda_{i}, i=1,2,3,4$, be chosen such that $\overline{\bigcup_{i=1}^{4} \Omega_{0}^{i}}$ cover $\Omega$. From (6.1) and (6.5) we have for $h$ sufficiently small and $i=1,2,3,4$

$$
\left\|U-K_{h} * U_{h}\right\|_{0, \Omega_{0}^{i}} \leqslant C\left(h^{2 r-2}\|U\|_{2 r-2, \Lambda_{i}}+\left\|U-U_{h}\right\|_{2-r, \Lambda_{i}}\right)
$$

and

$$
\left|U-K_{h} * U_{h}\right|_{0, \Omega_{0}^{i}} \leqslant C\left(h^{2 r-2}\|U\|_{2 r, \Lambda_{i}}+\left\|U-U_{h}\right\|_{2-r, \Omega} E\right) .
$$

It was shown in [15] that

$$
\left\|U-U_{h}\right\|_{2-r, \Lambda_{i}} \leqslant C h^{2 r-2}\|U\|_{r, \Lambda_{i}}
$$

The inequalities (7.8) and (7.9) now easily follow from (7.12), (7.13), (7.14), (7.11) and the fact that $\bigcup_{i=1}^{4} \Omega_{0}^{i}$ covers $\Omega$.

It remains to prove (7.10). We shall do so in the case $i=1$, the other cases follow in the same manner. Let $\Lambda=\Omega \cup \Omega_{1} \cup \Gamma_{1}$. We shall first show that

$$
\sum_{i=1}^{2} \int_{\Lambda} \frac{\partial\left(U-U_{h}\right)}{\partial x_{i}} \frac{\partial \varphi}{\partial x_{i}} d x=0, \quad \forall \varphi \in \bar{M}_{k, r}^{h}(\Lambda)
$$

If $\varphi \in \bar{M}_{k, r}^{h}(\Lambda)$, then let $\varphi=\varphi^{0}+\varphi^{e}$ where

$$
\varphi^{0}\left(x_{1}, x_{2}\right)=\frac{\varphi\left(x_{1}, x_{2}\right)-\varphi\left(-x_{1}, x_{2}\right)}{2}
$$

and

$$
\varphi^{e}\left(x_{1}, x_{2}\right)=\frac{\varphi\left(x_{1}, x_{2}\right)+\varphi\left(-x_{1}, x_{2}\right)}{2} .
$$

Now $\varphi^{0} \in \bar{M}_{k, r}^{h}\left(\Omega_{1}\right)$ and $\bar{M}_{k, r}^{h}(\Omega)$, hence using (7.7)

$$
\sum_{i=1}^{2} \int_{\Lambda} \frac{\partial\left(U-U_{h}\right)}{\partial x_{i}} \frac{\partial \varphi^{0}}{\partial x_{i}} d x=2 \sum_{i=1}^{2} \int_{\Omega} \frac{\partial\left(U-U_{h}\right)}{\partial x_{i}} \frac{\partial \varphi^{0}}{\partial x} d x=0 .
$$

Since $\varphi^{e}$ is an even function of $x_{1}$ and $U-U_{h}$ is an odd function of $x_{1}$ it follows that

$$
\sum_{i=1}^{2} \int_{\Lambda} \frac{\partial\left(U-U_{h}\right)}{\partial x_{i}} \frac{\partial \varphi^{e}}{\partial x_{i}} d x=0
$$

which proves (7.15).

Since $U$ and $U_{h}$ on $\Omega_{2}$ and $\Omega_{3}$ may be obtained from $U$ and $U_{h}$ on $\Lambda$ by an odd reflection about the line $y=1$, the argument given to prove (7.15) also shows that (7.10) holds which completes the proof.

Appendix. Here we present two tables. Table 1 gives values for the weights $k_{j}^{\prime}$ for various values of $r$. For a given choice of basis functions these may be used to calculate $K_{h} * u_{h}(x)$ at each point $x$ or just at mesh points using (5.8). In Table 2 we give values of the weights $d_{j}^{\prime}$ for various values of $r$ in the case where $S_{h}^{r}$ is chosen to be the splines generated by the $B$-spline basis $\psi^{(r)}$. 
TABLE 1. $k_{j}^{\prime}, l=r-2, t=r-1$

\begin{tabular}{ccrrr}
\hline$j \backslash \boldsymbol{r}$, & 3 & \multicolumn{1}{c}{4} & \multicolumn{1}{c}{5} & \multicolumn{1}{c}{6} \\
\hline 0 & $13 / 12$ & $37 / 30$ & $346517 / 241920$ & $76691 / 45360$ \\
1 & $-1 / 24$ & $-23 / 180$ & $-81329 / 322560$ & $-48061 / 113400$ \\
2 & & $1 / 90$ & $6337 / 161280$ & $20701 / 226800$ \\
3 & & & $-3229 / 967680$ & $-1573 / 113400$ \\
4 & & & & $479 / 453600$ \\
\hline
\end{tabular}

TABLE $2 . \quad d_{j}^{\prime}$

\begin{tabular}{|c|c|c|c|c|}
\hline$j \backslash r$ & 3 & 4 & 5 & 6 \\
\hline 0 & $51 / 72$ & $673 / 1080$ & $\frac{33055739}{58060800}$ & $\frac{967,356,037}{1,828,915,200}$ \\
\hline 1 & $11 / 72$ & $4283 / 21600$ & $\frac{3589969}{16257024}$ & $\frac{3,841,481,473}{16,460,236,800}$ \\
\hline 2 & $-1 / 144$ & $-61 / 5400$ & $\frac{-12162977}{1625702400}$ & $\frac{31,253,191}{82,301,184,000}$ \\
\hline 3 & & $29 / 21600$ & $\frac{4795283}{2438553600}$ & $\frac{48,179,483}{27,433,728,000}$ \\
\hline 4 & & $1 / 10800$ & $\frac{26273}{270950400}$ & $\frac{89711}{514,382,400}$ \\
\hline 5 & & & $\frac{-58243}{812851200}$ & $\frac{-2905789}{16,460,236,800}$ \\
\hline 6 & & & $\frac{-3229}{4877107200}$ & $\frac{25867}{1,097,349,120}$ \\
\hline 7 & & & & $\frac{117083}{82,301,184,000}$ \\
\hline 8 & & & & $479 / 164,602,368,000$ \\
\hline
\end{tabular}

Department of Mathematics

Cornell University

Ithaca, New York 14853

1. I. BABUŠKA, "The finite element method with Lagrangian multipliers," Numer. Math., v. 20, 1973, pp. 179-192. MR 50 \#11806.

2. J. BRAMBLE, "A survey of some finite element methods proposed for treating the Dirichlet problem," Advances in Math., v. 16, 1975, pp. 187-196.

3. J. H. BRAMBLE \& S. HILBERT, "Bounds for a class of linear functionals with applications to Hermite interpolation," Numer. Math., v. 16, 1971 , pp. 362-369.

4. J. H. BRAMBLE, J. A. NITSCHE \& A. H. SCHATZ, "Maximum-norm interior estimates for Ritz-Galerkin methods," Math. Comp., v. 29, 1975, pp. 677-688. 
5. J. H. BRAMBLE \& J. E. OSBORNE, "Rate of convergence estimates for nonselfadjoint eigenvalue approximations," Math. Comp., v. 27, 1973, pp. 525-549. MR 51 \#2280.

6. J. H. BRAMBLE \& A. H. SCHATZ, "Estimates for spline projection," Rev. Française Automat. Informat. Recherche Opérationnelle Analyse Numérique, v. 10, n 8, août 1976, pp. 5-37.

7. J. H. BRAMBLE \& A. H. SCHATZ, "Higher order local accuracy by averaging in the tinite element method,"Mathematical Aspects of Finite Elements in Partial Differential Equations (Proc. Sympos., Math. Res. Center, Univ. of Wisconsin, Madison, 1974), edited by Carl de Boor, Academic Press, New York, 1974, pp. 1-14. MR 50 \#1525.

8. J. H. BRAMBLE \& M. ZLÁMAL, "Triangular elements in the finite element method," Math. Comp., v. 24, 1970, pp. 809-820. MR 43 \#8250.

9. C. de BOOR \& B. SWARTZ, "Collocation at Gaussian points," SIAM J. Numer. Anal., v. 10, 1973, pp. 582-606. MR 51 \#9528.

10. J. DOUGLAS, JR. \& T. DUPONT, "Some superconvergence results for Galerkin methods for the approximate solution of two point boundary problems." (Preprint.)

11. J. DOUGLAS, JR., T. DUPONT \& M. F. WHEELER, "Some super-convergence results for an $H^{1}$-Galerkin procedure for the heat equation," Computing Methods in Applied Sciences and Engineering. Part I (Proc. Internat. Sympos., Versailles, 1973), Springer-Verlag, Berlin and New York, 1974, pp. 288-311. MR 49 \#4197.

12. A. FRIEDMAN, Partial Differential Equations, Holt, Rinehart and Winston, New York, 1969.

13. J. L. LIONS \& E. MAGENES, Non-Homogeneous Boundary Value Problems and Applications. Vol. I, Springer-Verlag, Berlin and New York, 1972. MR 50 \#2670.

14. J. A. NITSCHE, “Über ein Variationsprinzip zur Lösung von Dirichlet-Problemen bei Verwendung von Teilräumen, die keinen Randbedingungen unterworfen sind,"Abh. Math. Sem. Univ. Hamburg, v. 36, 1971 , pp. 9-15. MR 49 \#6649.

15. J. A. NITSCHE \& A. H. SCHATZ, "Interior estimates for Ritz-Galerkin methods," Math. Comp., v. 28, 1974, pp. 937-958. MR 51 \#9525.

16. I. J. SCHOENBERG, "Contributions to the problem of approximation of equidistant data by analy tic functions," Parts A, B, Quart. Appl. Math.,v. 4, 1946, pp. 45-99, 112-141. MR 7, 487; 8, 55.

17. V. THOMÉE, "Spline approximation and difference schemes for the heat equation," The Mathematical Foundations of the Finite Element Method with Applications to Partial Differential Equations (Proc. Sympos., Univ. of Maryland, 1972), edited by A. K. Aziz, Academic Press, New York, 1972, pp. $711-746$. MR 49 \#11824.

18. V. THOMÉE \& B. WENDROFF, "Convergence estimates for Galerkin methods for variable coefficient initial value problems," SIAM J. Numer. Anal., v. 11, 1974, pp. 1059-1068. MR 51 \#7309. 